\title{
Un récit mobilisateur pour la jeunesse : les initiatives des institutions européennes
}

\section{Armand Beuf}

\section{(2) OpenEdition}

1 Journals

Édition électronique

URL : https://journals.openedition.org/ries/11240

DOI : 10.4000/ries. 11240

ISSN : 2261-4265

Éditeur

France Education international

\section{Édition imprimée}

Date de publication : 1 décembre 2021

Pagination : 22-25

ISBN : 978-2-85420-637-9

ISSN : $1254-4590$

\section{Référence électronique}

Armand Beuf, « Un récit mobilisateur pour la jeunesse : les initiatives des institutions européennes », Revue internationale d'éducation de Sèvres [En ligne], 88 | décembre 2021, mis en ligne le 01 décembre 2022, consulté le 26 mars 2023. URL : http://journals.openedition.org/ries/11240 ; DOI : https:// doi.org/10.4000/ries.11240

Ce document a été généré automatiquement le 26 mars 2023.

Tous droits réservés 


\title{
Un récit mobilisateur pour la jeunesse : les initiatives des institutions européennes
}

\author{
Armand Beuf
}

1 En 2017, l'Union européenne (UE) comptait 88 millions de jeunes âgés de 15 à 29 ans, soit $17 \%$ de sa population. À l'appui du renforcement de la gouvernance pour promouvoir la participation de jeunes européens aux processus décisionnels, qui est souhaitée au sein du Conseil des ministres de l'UE ${ }^{1}$, des spécialistes de l'éducation ainsi que des institutions européennes (Commission européenne ${ }^{2}$, Parlement européen) ou consultatives (Comité économique et social européen: CESE) recommandent l'élaboration et le partage de récits mobilisateurs pour susciter chez les jeunes européens de nouvelles attitudes plus responsables en matière d'écocitoyenneté et plus attractives en matière d'apprentissage tout au long de leur vie. La conférence sur l'avenir de l'Europe constitue un moment privilégié de partage d'un tel récit, qui peut mobiliser et façonner l'imaginaire européen. Cette conférence, qui se déroule jusqu'au printemps 2022, offre une occasion privilégiée de débattre des priorités de l'Europe et des défis auxquels l'ensemble des citoyens, et notamment les jeunes, est confronté et de parvenir à dégager des orientations possibles pour y faire face.

[L]e succès des travaux de l'actuelle conférence sur le futur de l'Europe dépendra notamment de sa capacité à définir un nouveau récit pour l'Europe ancré dans les réalités de la vie quotidienne, et que les citoyens européens pourront s'approprier. [...] L'objectif est de redécouvrir et de renouveler un sentiment indispensable de communauté fondé sur des valeurs communes, en insufflant à l'Europe une nouvelle dynamique pour relever les défis actuels et futurs ${ }^{3}$.

\section{Un récit mobilisateur dans la continuité de nombreuses initiatives antérieures}

Les programmes Comett (1986), Erasmus (1987), Jeunesse pour l'Europe (1989), Leonardo Da Vinci (1995), le service volontaire européen (1996), Grundtvig (2000), 
Erasmus Mundus (2004) et Erasmus entrepreneurs (2009), renforcés au fil du temps par des consultations publiques comme le livre blanc ${ }^{4}$ proposé en novembre 2001 par la Commission européenne pour un nouvel élan de la jeunesse européenne, ont constitué des investissements à long terme pour construire et rendre crédible un tel récit mobilisateur en direction des jeunes.

3 Sur les trente dernières années et pour les trente-trois pays participants, les nombres de bénéficiaires sont importants : 4400000 étudiants de l'enseignement supérieur entre 1987 et 2017; 1300000 jeunes de l'enseignement et de la formation professionnels impliqués dans les échanges de jeunes depuis 1988; 1800000 enseignants, formateurs et travailleurs du secteur de la jeunesse depuis 1989; 100000 jeunes dans le service volontaire européen depuis 1996 et 100000 étudiants et enseignants dans les coopérations avec des universités hors d'Europe (depuis 2004)5.

4 Autre résultat significatif : Le programme Erasmus entrepreneurs ouvert aux jeunes entrepreneurs à partir de 2009 a, quant à lui, renforcé l'esprit d'entreprise, suscité la création de nouvelles entreprises et de nouveaux emplois ainsi que l'ouverture de nouveaux marchés depuis onze ans.

5 Les acquis de la préservation de l'environnement européen sont un autre exemple significatif à souligner pour les jeunes générations, avec les huit programmes d'action successifs pour l'environnement européen et la création de sites naturels ou seminaturels dénommés « Natura 2000 », présentant une grande valeur patrimoniale par la faune et la flore exceptionnelles qu'ils comprennent. Le principe de précaution a été retenu et celui du pollueur payeur a été établi grâce à la détermination du commissaire européen à l'environnement Lorenzo Natali, dans la décennie 1970. Un premier programme de recherche et développement de climatologie a été lancé en 1985 et le traité d'Amsterdam, conclu en 1997, a intégré la protection de l'environnement dans toutes les politiques sectorielles de l'UE.

6 De plus, en matière de recherche et d'innovation (R\&I), neuf programmes-cadres, de 1987 à nos jours, ont permis de doter l'Union européenne de solides écosystèmes de R\&I composés de réseaux d'excellence de chercheurs confirmés et de jeunes chercheurs, de constituer des consortiums de R\&I consolidant le marché intérieur européen et ses PME et renforçant la coopération scientifique internationale ${ }^{6}$ de l'UE avec ses partenaires associés.

\section{Un récit mobilisateur: des initiatives récentes dans la continuité}

7 Ainsi, l'association des experts et des évaluateurs de l'Union européenne (EvalUE) a décidé, en septembre 2020, de soutenir l'objectif de création de l'espace européen d'éducation à l'horizon 2025 et de demande de crédits supplémentaires pour sa mise en œuvre. Devant la déscolarisation causée par la pandémie de Covid-19, elle a décidé de devenir membre de la Coalition globale pour l'éducation créée par l'Unesco ${ }^{7}$ en 2020 . Ce partenariat lui permet de proposer à un cercle élargi d'acteurs la formulation de nouveaux objectifs politiques en matière d'éducation des jeunes apprenants, la caractérisation de modèles plus inclusifs et équitables, l'exploration de nouvelles façons d'apprendre avec le numérique, tout en proposant un projet de charte sur la protection des droits d'utilisateurs d'internet et des réseaux sociaux, l'exploration de 
nouveaux modes de gouvernance et de pilotage des établissements d'enseignement et des modalités d'évaluation des systèmes éducatifs, de même qu'une réflexion sur l'apprentissage tout au long de la vie, qui reste une priorité stratégique ${ }^{8}$ de l'Union.

Les négociations interétatiques et interinstitutionnelles européennes de l'automne 2020 ont permis de sauvegarder l'objectif de création de cet espace européen de l'éducation et les États membres ont fait connaître leurs orientations en matière d'éducation et de formation pour 2021-2030, dans leur résolution adoptée en février dernier. Cet objectif ambitieux et de long terme concerne aussi les jeunes citoyens. Pourquoi ne pas saisir cette opportunité pour mettre l'accent sur la dimension européenne dans l'intitulé de chacun des ministères chargés de l'éducation nationale? Les politiques et programmes européens deviendraient ainsi des composants du cursus de base de l'élève ou de l'étudiant européen au cœur même de la diversité des systèmes éducatifs nationaux.

9 N'oublions pas également la capacité de la jeunesse d'influer sur l'élaboration et le développement des sciences citoyennes qualifiées aussi de participatives 9 . Les jeunes deviennent en effet de plus en plus des acteurs de la mise en œuvre de ces programmes participatifs sur plusieurs continents, en développant leurs compétences d'observation et en mobilisant une grande capacité de traitement de données scientifiques collectées, ce qui constitue un atout indéniable de développement de ces sciences. Le soutien d'organisations internationales, telles que l'Unesco, ou régionales, comme l'Union européenne, à la science ouverte est particulièrement utile pour accélérer la reconnaissance de leur rôle de " capteurs sociaux ".

10 Associer la jeunesse au développement économique régional est particulièrement important, comme le montrent les exemples suivants. Ainsi, l'un des cinq axes fondateurs du parc régional finlandais Espoo vise l'inclusion des jeunes dans son dispositif d'orientation stratégique ${ }^{10}$. De même, des régions impliquent leurs citoyens dans le choix de leur stratégie de spécialisation intelligente : la Lombardie, l'une des régions les plus industrialisées, a par exemple organisé des consultations publiques citoyennes pour définir des choix prioritaires d'investissement de son écosystème de R\&I. Les jeunes sont une cible privilégiée de ces campagnes de consultation que les autorités régionales sont à même de multiplier.

11 Enfin, le programme pour l'Europe numérique doit prévoir d'associer des jeunes à l'implantation future des pôles européens d'innovation numérique et à la conception des dispositifs de formation. La multiplication de magazines participatifs numériques écrits par des jeunes pour des jeunes, comme Cafébabel ${ }^{11}$ a pour vocation d'attirer de nombreux jeunes aux réalités européennes.

Lors du discours sur l'état de l'Union européenne en 2021, la présidente de la Commission européenne a déclaré 2022 "année de la jeunesse ». Gageons que cette jeunesse "déterminée " approfondira sa vision du futur grâce au dialogue avec l'ensemble des partenaires engagés dans la transmission intergénérationnelle mise en exergue par Stéphane Hessel dans un ouvrage intitulé Engagez-vous. 


\section{NOTES}

1. https://bit.ly/3qPN97F

2. Communication de la Commission européenne, 25 mai 2018 : Mobiliser, connecter et autonomiser les jeunes : une nouvelle stratégie de l'UE en faveur de la jeunesse. https://bit.ly/30GSJyv

3. Avis du CESE, 27 avril 2021 : Un nouveau récit pour l'Europe. https://bit.ly/3Hwq9At

4. https://bit.ly/3Hz6gJ4

5. J.-.P. Bobichon (2021) : «Erasmus et Erasmus+. Origines et impacts?», AIACE Vox, avril. https://www.aiacefr.eu/images/Publications/VOX\%20118_WEB.pdf

6. A. Beuf (2010). «Enhancing European partnerships for international scientific and technological cooperation ». In D. T. Mihailovic et B. Lalic (eds), Advances in environmental modelling and measurements. New York : Nova publishers, p. 1-11.

7. https://fr.unesco.org/covid19/educationresponse/globalcoalition

8. Rapport du groupe d'experts d'EvalUE préparé pour l'Unesco dans le cadre de la Coalition mondiale de l'éducation: Éducation au futur: nouveaux défis, nouvelles réponses. https://bit.ly/ 3oJEHEn

9. Les sciences citoyennes ou participatives ont trait, par exemple, à l'étude de la biodiversité, à l'astronomie ; les citoyens peuvent y participer pour la mise au point de protocoles d'observation et de traitement des données collectées.

10. S. Hertell (2016). Espoo innovation garden in regional innovations sytems. https://bit.ly/3Fy5MB0

11. https://cafebabel.com/fr/

\section{INDEX}

Palabras claves : joven, programas europeos, espacio europeo de educación, ciudadanía

Keywords : youth, European programmes, European education area, citizenship

Mots-clés : jeune, programmes européens, espace européen d'éducation, citoyenneté

Index géographique : Europe

\section{AUTEUR}

\section{ARMAND BEUF}

Armand Beuf a coordonné le rapport Éducation au futur : nouveaux défis, nouvelles réponses du groupe d'experts de l'association des experts et évaluateurs de l'UE (EvalUE), partenaire de la coalition globale pour l'éducation lancée par l'Unesco en 2020. Ancien membre du Comité directeur de formation de la Commission européenne, il a exercé comme formateur d'adultes de nombreuses années. Courriel : armandbeuf[at]hotmail.com 